\section{Autism DNA hub opens}

WuXi NextCODE, based in Cambridge, Massachusetts, and the New York-based Simons Foundation in March announced they would jointly make the world's largest autism dataset, the Simons Simplex Collection (SSC), available to researchers online. This collaboration is a step towards enabling researchers to use the internet to make large-scale genome comparisons. The SSC, part of the Simons Foundation Autism Research Initiative (SFARI), is a permanent repository of genetic samples comprising 10,000 exomes from 2,600 so-called 'simplex' families, where one child is affected by an autism spectrum disorder, whereas parents and siblings are unaffected. The repository is already accessible to autism researchers from 17 leading institutions from the US, Canada, China, France, Iceland, Austria, Ireland, Brazil and Qatar, but with the new initiative, researchers worldwide can apply for access to the company's cloud-based database, the WuXi NextCODE Exchange. The SSC can now serve as the hub of an ever-growing network of autism datasets, said Louis Reichardt, SFARI's director, in a company release. The partnership is a way for big genomics to accelerate the understanding of autism and other complex conditions, said Timothy Yu, a clinician and assistant professor of neurology at Boston Children's Hospital. "This is a game-changer, and we are already using the WuXi NextCODE SSC portal to validate and extend new discoveries and confirm clinical diagnoses," said Yu.

\section{6 "This is the kind of news CIRM has yearned for in recent years: significant private cash that gives CIRM-funded projects a push to become actual products and to return value to the state," writes journalist Alex Lash about Stanford spin-out Forty Seven (named after the cell surface marker CD47). The project, out of Irving Weissman's lab, received $\$ 30$ million for work on CD47 antibodies; the university received over $\$ 300$ million, including funds to build a stem cell research center, headed by Weissman. (Xconomy, 25 February 2016)}

"At Kelkoo, we needed to connect shoppers with retailers. At Kayak, you connect travelers with airlines. And here [clinical trials], it's exactly the same thing: you have patients and researchers. I asked, 'So why is no one connecting them?'" Argentinian entrepreneur Pablo Graiver, who founded airfare search engine Kayak, asked, and has now designed a new tool, http://www. trialreach.com/, to help patients wanting to find out about clinical trials connect with companies testing them. (Bloomberg Business, 11 March 2016)

"Gleevec is an amazing drug but it put us down the completely wrong path," says @ DrPatSoonShiong, arguing against targeted therapy. \#FOGM16
"The business that I am running is growing at $100 \%$ year over year, and it's only two years old," says Nathan Wood, president of SGI-DNA, the DNA synthesis arm of the La Jolla, California-based Synthetic Genomics. SGI-DNA also anticipates a year of continued growth in 2016, not only among its industrial accounts, but also with academics, some of whom are bringing SGI-DNA's do-it-yourself gene synthesis instrument, the BioXP 3200, which retails at nearly $\$ 60,000$, in house. But at $\$ 0.15$ per base pair for sequences up to $2 \mathrm{~kb}$, many choose to leave the job of DNA synthesis to the company.

So far, DNA synthesis has stirred the most interest among industrial players. In November, Twist announced an agreement with Ginkgo Bioworks, a Boston-based synthetic biology company that engineers new microorganisms for industrial uses such as producing synthetic sweeteners, cosmetics, biofuels and probiotics. Twist will supply Ginkgo with a 100 million base pairs of synthetic DNA over the year-a deal that represents $10 \%$ of the 2015 global DNA synthesis market, according to the companies.

Ginkgo co-founder Barry Canton says the company has been buying DNA from different suppliers for eight years, but now, Twist's $\$ 0.10$ per base pair represents a tenfold decrease from the $\$ 1$ per base pair in 2005 . Back then, it cost $\$ 1,000$ to synthesize a gene, whereas now it will cost $\$ 100$. "The next milestone is a cent per base pair," Canton forecasts. "At that point, a gene could cost in the range of $\$ 10$."

These improvements in pricing, turnaround time, length and fidelity are happening across the DNA synthesis market, notes Andy May, CSO at Caribou Biosciences, a Berkeley, California-based company that offers gene engineering services for researchers in academia, pharma, agbiotech and other fields. Caribou buys its DNA from a number of providers, including Integrated DNA Technologies, an established manufacturer of custom nucleic acids. Based in Coralville, Iowa, IDT advertises custom gene synthesis priced at $\$ 0.30$ per base pair and turnaround times between eight days and three weeks, depending on the order. The company also relies on mass spectrometry and sequencing for quality control analysis.

For Andrew Hessel, a genetic engineer at Autodesk Research, a San Francisco-based company that makes DNA origami nanoparticles and whole-viral genome constructs aimed at personalized cancer treatments or new vaccines, the forecasted growth in the gene synthesis market depends closely on advances in synthetic biology methods. "For the last few years, the software design tools have been advancing faster than the ability to print DNA," he says. "Now synthesis is catching up."

That may be so, but the market is still considered to be nascent, and the comparisons to Illumina 2.0 may be premature, notes Rob Carlson, an industry observer at Seattlebased consultancy Biodesic. "I don't think writing for biological engineering will ever approach reading," Carlson says. "As the price per base falls, it is hard to see how the demand for bases can grow fast enough to increase the dollar value of the market very quickly," he adds. "Reading, on the other hand, will be done in more instances and more frequently."

DNA storage applications offer another avenue for growth. Synthetic DNA suppliers will have to make a lot of DNA, however, to compete with archival tape. Carlson calculates that, to compete with tape, a DNA drive would have to be approximately six orders of magnitude higher throughput than anything commercially available today and about five orders of magnitude lower in cost per base. But as data production soars, the need for a high-density storage will ramp up. "It isn't actually clear how tape or disks can do the job," says Carlson, "and right now DNA is the only thing that passes the bar."

Endy, a synthetic biologist and professor of bioengineering, agrees with Carlson that data storage is a "huge market" for DNA synthesis firms in terms of size, and says it's potentially easy to supply in terms of fabrication. Moreover, he says it is "absolutely essential" that "nontraditional biotech markets" such as DNA storage, get established for commercial DNA construction platforms.

At the same time, Endy believes that it will take further technology innovation for the market to reach its true potential. He says the industry needs to move away from the surface-based oligo synthesis techniques used by many major players to alternative, enzymatic technologies, such as those being commercialized by San Diego-based Molecular Assemblies, which eschew the chemicals used in traditional synthesis for natural enzymes, improving the quality of the DNA produced.

Such methods could support DNA printers that "might be able to print an entire genome in minutes," says Endy, with no assembly required. "Stated differently, anyone who thinks that current practice at Gen9 [and other companies] represents the final state of the art needs to think again." A few cents per base pair is "not a milestone," he adds. "It's just another number."

Justin Petrone Tartu, Estonia 